\title{
A NOTE ON LONGEST PATHS IN CIRCULAR ARC GRAPHS
}

\author{
FELIX Joos \\ Institut für Optimierung und Operations Research \\ Universität Ulm, Ulm, Germany \\ e-mail: felix.joos@uni-ulm.de
}

\begin{abstract}
As observed by Rautenbach and Sereni [SIAM J. Discrete Math. 28 (2014) 335-341] there is a gap in the proof of the theorem of Balister et al. [Combin. Probab. Comput. 13 (2004) 311-317], which states that the intersection of all longest paths in a connected circular arc graph is nonempty. In this paper we close this gap.
\end{abstract}

Keywords: circular arc graphs, longest paths intersection.

2010 Mathematics Subject Classification: 05C38.

\section{REFERENCES}

[1] P.N. Balister, E. Győri, J. Lehel and R.H. Schelp, Longest paths in circular arc graphs, Combin. Probab. Comput. 13 (2004) 311-317. doi:10.1017/S0963548304006145

[2] T. Gallai, Problem 4, in: Theory of graphs, Proceedings of the Colloquium held at Tihany, Hungary, September, 1966,. P. Erdős and G. Katona Eds., Academic Press, New York-London; Akadmiai Kiad, Budapest (1968).

[3] J.M. Keil, Finding Hamiltonian circuits in interval graphs, Inform. Process. Lett. 20 (1985) 201-206. doi:10.1016/0020-0190(85)90050-X

[4] D. Rautenbach and J.-S. Sereni, Transversals of longest paths and cycles, SIAM J. Discrete Math. 28 (2014) 335-341. doi:10.1137/130910658

[5] A. Shabbira, C.T. Zamfirescu and T.I. Zamfirescu, Intersecting longest paths and longest cycles: A survey, Electron. J. Graph Theory Appl. 1 (2013) 56-76. doi:10.5614/ejgta.2013.1.1.6 